\title{
Fixation disengagement and eye-movement latency
}

\author{
WA JAMES TAM and HIROSHI ONO \\ York University, North York, Ontario, Canada
}

\begin{abstract}
We examined eye-movement latencies to a target that appeared during visual fixation of a stationary stimulus, a moving stimulus, or an extrafoveal stimulus. The stimulus at fixation was turned off either before target onset (gap condition) or after target onset (overlap condition). Consistent with previous research, saccadic latencies were shorter in gap conditions than they were in overlap conditions (the gap effect). In Experiment 1, a gap effect was observed for vergence eye movements. In Experiment 2, a gap effect was observed for saccades directed at a target that appeared during visual pursuit of a moving stimulus. In Experiment 3, a gap effect was observed for saccades directed at a target that appeared during extrafoveal fixation. The present results extend reports of the gap effect for saccadic shifts during visual fixation to (a) vergence shifts during visual fixation, (b) saccadic shifts during smooth visual pursuit, and (c) saccadic shifts during extrafoveal fixation. The present findings are discussed with respect to the incompatible goals of fixation-locking and fixation-shifting oculomotor responses.
\end{abstract}

Saccadic latency to the onset of a target has been shown to depend on the temporal interval between target onset and the termination of the stimulus at fixation (e.g., Becker, 1972; Cohen \& Ross, 1977; Iwasaki, 1990; Mayfrank, Mobashery, Kimmig, \& Fischer, 1986; Reulen, 1984; L. E. Ross \& S. M. Ross, 1980; S. M. Ross \& L. E. Ross, 1981, 1983; Saslow, 1967). Latencies are shorter when the stimulus at fixation is extinguished before target onset (gap condition) than they are when it is extinguished after target onset (overlap condition). This effect of stimulus offset on saccadic latencies is referred to as the gap effect. The effect is important in research on oculomotor behavior because of its relevance to processes involved in the preparation of eye movements. The goal of the present study was to extend the domain of study of the gap effect by investigating latencies of vergence eye movements and by examining latencies of saccadic eye movements while the eyes were pursuing a moving stimulus or fixating an extrafoveal stimulus.

Recent studies of the gap effect have concentrated on the role of attentional processes, assuming that the gap

Some of the work reported here was based on a doctoral dissertation by the first author and was presented in 1988 at the annual meeting of the Association for Research in Vision and Ophthalmology. The work was supported by Grant A0296 awarded to H.O. by the Natural Sciences and Engineering Research Council of Canada. We thank Paul Hearty and Lew Stelmach for their support in writing the paper while the first author was a postdoctoral fellow at the Communications Research Centre, Canada. We particularly thank Lew Stelmach for his helpful comments on an earlier version of the paper. Correspondence concerning this article may be addressed to J. Tam, Communications Research Centre, 3701 Carling Avenue, Ottawa, ON, Canada K2H 8S2 (e-mail: james@dgbt.doc.ca). effect is due to facilitation of attentional disengagement before a saccade (e.g., Braun \& Breitmeyer, 1988; Fischer \& Breitmeyer, 1987; Fischer \& Weber, 1993). According to this view, saccadic latencies are shorter in gap conditions than they are in overlap conditions because extinguishing the fixation stimulus leads to facilitation of attentional disengagement (e.g., Braun \& Breitmeyer, 1988; Fischer, 1987; Fischer \& Breitmeyer, 1987; Fischer \& Weber, 1993; Mayfrank et al., 1986). In our view, however, attentional processes play little or no role in explaining the gap effect, because saccadic latencies can be shorter in gap conditions even when attentional disengagement is unlikely to have occurred (Kingstone \& Klein, 1993a; Tam \& Stelmach, 1993). In experiments in which allocation of attention was measured, the gap effect appeared to depend more on whether the foveated stimulus was extinguished than on whether an attended stimulus was extinguished. In other words, the critical event influencing the gap effect was the offset of the fixated stimulus, irrespective of the direction of attention (Kingstone \& Klein, 1993a; Klein, Kingstone, \& Pontefract, 1992; Tam \& Stelmach, 1993).

The gap effect is more aptly explained using oculomotor rather than attentional processes (Kingstone \& Klein, 1993a, 1993b; Reuter-Lorenz, Hughes, \& Fendrich, 1991; Tam \& Stelmach, 1993). Extinguishing the fixation stimulus facilitates saccadic latencies by breaking the feedback loop that maintains the eye directed at the fixation stimulus, thereby freeing the eye to move to a new target. This explanation assumes that during fixation the oculomotor system is effectively locked or engaged on the fixation target and that voluntary shifts of fixation and reflexive glances toward novel objects are inhibited (Guitton, Buchtel, \& Douglas, 1985; Lynch, Mountcastle, Talbot, \& Yin, 1977; Marocco, 1978). Be- 
fore a shift in fixation can occur, unlocking or disengagement must take place.

By varying the asynchrony between the offset of the fixation stimulus and the onset of the target stimulus, it is possible to control the state of the oculomotor system at the moment of target onset. In gap conditions, the stimulus at fixation is turned off before the onset of the target. If the duration of the gap between fixation stimulus offset and target onset is large (e.g., $200 \mathrm{msec}$ ), the oculomotor system will have become completely disengaged and will be in an optimal state to respond to the onset of the target. If the duration of the gap is short (e.g., $50 \mathrm{msec}$ ), disengagement will be only partially completed when the target appears, and saccadic latencies will be slightly longer. If the fixation stimulus is still present when the target appears-that is, if it overlaps in time with the target stimulus-disengagement will not have occurred, and latencies will be longest.

To date, studies of the gap effect have focused on saccadic latencies during foveal fixation of a stationary stimulus (e.g., Becker, 1972; Cohen \& Ross, 1977; Fischer \& Ramsperger, 1984; Iwasaki, 1990; Reulen, 1984; Reuter-Lorenz et al., 1991; L. E. Ross \& S. M. Ross, 1980; S. M. Ross \& L. E. Ross, 1981, 1983; Saslow, 1967). The goal of the present research was to explore the gap effect using different types of eye movements and a richer set of stimuli. In Experiment 1, we measured latencies of vergence eye movements to targets that appeared at different temporal intervals with respect to the termination of a fixated stimulus. In Experiment 2, we measured latencies of saccades to targets that appeared during smooth pursuit of a moving stimulus; the pursued stimulus was either turned off or left on when the target appeared. Finally, in Experiment 3, we measured latencies of saccades to targets that appeared during fixation of an extrafoveal stimulus; the extrafoveal stimulus was turned off either before or after target onset.

\section{EXPERIMENT 1}

The purpose of this experiment was to determine whether a gap effect would generalize to vergence eye movements. We measured latencies of vergence eye movements to a target that was positioned at a different depth from an initially fixated stimulus. The target could appear in front of or behind the fixated stimulus. The subjects' task was to execute a vergence eye movement to the target.

On the basis of the oculomotor disengagement hypothesis, one might expect that, in comparison with overlap conditions, latencies of vergence eye movements would be reduced in gap conditions. In overlap conditions, the oculomotor system would be engaged in maintaining the eyes directed at the fixation stimulus. In contrast, in gap conditions, the offset of the fixation stimulus would have facilitated disengagement. That is, the oculomotor system would be more ready to respond to the onset of the target in gap conditions than it would be in overlap conditions.
For comparison, latencies of saccades to the onset of a target located to the left and to the right of the initial fixation stimulus were also measured, replicating situations under which the gap effect is typically investigated.

\section{Method}

Subjects. Five subjects from the university community participated in the experiment. All except one were experienced in eyemovement experiments, and three were naive about the experimental hypothesis.

Stimuli. The initial fixation stimulus was a $3-\mathrm{mm}$-diameter light-emitting diode flanked by four smaller diodes (above, below, on the left, and on the right of the larger diode). This composite fixation stimulus subtended an angle of $37 \mathrm{~min}$ of arc at a viewing distance of $46.5 \mathrm{~cm}$. Targets were also 3 -mm-diameter lightemitting diodes. For vergence trials, one target diode was located in front of, and another behind, the initial fixation stimulus. For saccadic trials, one target diode was located on the left of the fixation stimulus, while another was located on the right. The targets were located such that each target diode and the initial fixation stimulus subtended an angle of $3^{\circ}$. For calibration, three lightemitting diodes were placed in a horizontal row at $4^{\circ}, 8^{\circ}$, and $12^{\circ}$, on both sides of the fixation stimulus.

Apparatus. A computer was used to control the offset and the onset of the fixation and target diodes. Eye positions were monitored with a Biometrics SGH/V-2 infrared limbic-tracker and the data were recorded onto magnetic tape with a Crown/Vetter type A FM recorder. The recordings were transferred onto paper using a Beckman type R Dynograph, with a paper speed of $50 \mathrm{~mm} / \mathrm{sec}$. The system had a bandwidth of $65 \mathrm{~Hz}$ and was able to measure horizontal eye movements linearly within $\pm 15^{\circ}$ with a resolution of $0.25^{\circ}$. A bite bar was used to minimize head movements.

Design. There were two factors in the experiment: (1) target position, at four levels: left or right for saccades, and near or far for vergence; and (2) offset-onset asynchrony, at six levels: -150 , $-75,0,75,150$, and $225 \mathrm{msec}$. Offset-onset asynchrony refers to the temporal interval between the offset of the initial fixation stimulus and the onset of the target. A negative offset-onset asynchrony indicates an overlap condition and a positive offset-onset asynchrony indicates a gap condition. A zero asynchrony indicates that the fixation stimulus was turned off at the same time as the target was turned on. Thus, there were 24 experimental conditions ( 4 target positions $\times 6$ offset-onset asynchronies).

Procedure. At the beginning of a trial, only the fixation stimulus was turned on. The subjects were instructed to fixate the stimulus and to shift fixation as quickly as possible to a target when it appeared. Each trial was initiated by the computer. After a foreperiod that varied randomly within a range of 1.5 to $2.5 \mathrm{sec}$, a target appeared at one of four possible locations: in front of, behind, to the left, or to the right of the initially fixated stimulus. Depending on the offset-onset asynchrony that was selected for a given trial, the fixation stimulus was turned off before, after, or at the same time as the onset of the target.

Each subject completed 30 trials per experimental condition. The trials were divided into five sessions, each held on a separate day. For each session, a subject completed six blocks of trials, with 24 trials per block. Target position and offset-onset asynchrony were randomized within each block.

\section{Results and Discussion}

The purpose of Experiment 1 was to explore the gap effect in the context of vergence eye movements. Figure 1 shows the mean latencies for vergence eye movements averaged over all five subjects; squares represent the data for divergence eye movements to the far target, while circles represent the data for convergence eye move- 


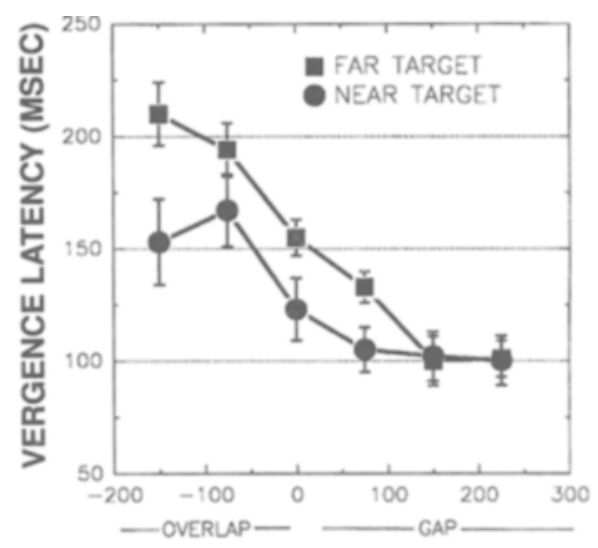

OFFSET-ONSET ASYNCHRONY (MSEC)

Figure 1. Vergence latency as a function of the interval between the termination of an initially fixated stimulus and the appearance of a target for a vergence movement. Each circle and square represents the mean latency of five subjects. Vertical bars through the data points are standard errors of the mean.

ments to the near target. For both divergence and convergence, latencies were shorter in gap conditions than they were in overlap conditions. Comparing latencies in the overlap condition of $-150 \mathrm{msec}$ and the gap condition of $225 \mathrm{msec}$, the mean reduction in latency for vergence eye movements was $80 \mathrm{msec}$.

The mean saccadic latencies averaged over all five subjects are shown in Figure 2. The reduction in saccadic latencies between the overlap condition of -150 $\mathrm{msec}$ and the gap condition of $225 \mathrm{msec}$ was $140 \mathrm{msec}$, indicating a gap effect. The absolute latency values were comparable to those obtained by previous investigators (e.g., Iwasaki, 1990; Saslow, 1967), except that in the $-150-\mathrm{msec}$ and $-100-\mathrm{msec}$ overlap conditions of the present experiment, latencies were longer by about $25-50 \mathrm{msec}$. This difference may be due to the fact that in the present experiment targets for saccades were intermixed with those for vergence, but were not in the other studies.

The most important finding of Experiment 1 was that the gap effect was observed equally well with vergence eye movements as it was with saccadic eye movements. In both cases, the effect of offset-onset asynchrony on latencies was statistically significant $[F(5,20)=34.65$, $p<.01$ for vergence latencies, and $F(5,20)=173.06, p<$ .01 for saccadic latencies]. These results suggest that the gap effect may be a general property of fixation-shifting eye movements. (For those interested in the distribution of eye-movement latencies, frequency distributions for one representative subject, for all 18 experimental conditions, are shown in Figure Al in the appendix.)

Although not central to the hypothesis under study, the interaction between target position and offset-onset asynchrony for vergence latencies was statistically significant $[F(5,20)=4.97, p<.01]$. As shown in Figure 1, vergence latencies to the near target (represented by cir- cles) were consistently shorter than were those to the far target (represented by squares). However, this difference was not found at an offset-onset asynchrony of $150 \mathrm{msec}$ and beyond. The latency difference between vergence to the near and far targets is consistent with previous reports of differences in the time course of convergence and divergence eye movements (e.g., Zuber \& Stark, 1968). The latency difference may be due to differences in retinal stimulation (the near target stimulated the temporal hemi-retinas, whereas the far target stimulated the nasal hemi-retinas); alternatively, it may be due to differences between the sets of muscles that were used for convergence and divergence. Whatever the reason for the difference, the important aspect of the findings was that the gap effect was observed for both convergent and divergent eye movements.

\section{EXPERIMENT 2}

To further explore the gap effect, we measured latencies of saccades to targets that appeared while the eyes were engaged in smooth pursuit. The goal of smooth pursuit is to maintain the image of a moving object on the fovea. Turning off the pursuit stimulus should facilitate disengagement by breaking the feedback loop that maintains the eyes locked on the fixation stimulus. In turn, this should lead to shorter latencies to a new target in gap conditions than in overlap conditions.

For practical reasons, the only "gap" condition that was implemented in the present experiment was the 0 -msec condition, in which the onset of the target was simultaneous with the offset of the pursuit stimulus; subjects were unable to maintain smooth pursuit reliably without a visible stimulus. In the overlap condition, the pursuit stimulus was left on until the end of the trial.

An additional condition was included in the present experiment, in which the subjects were required to exe-

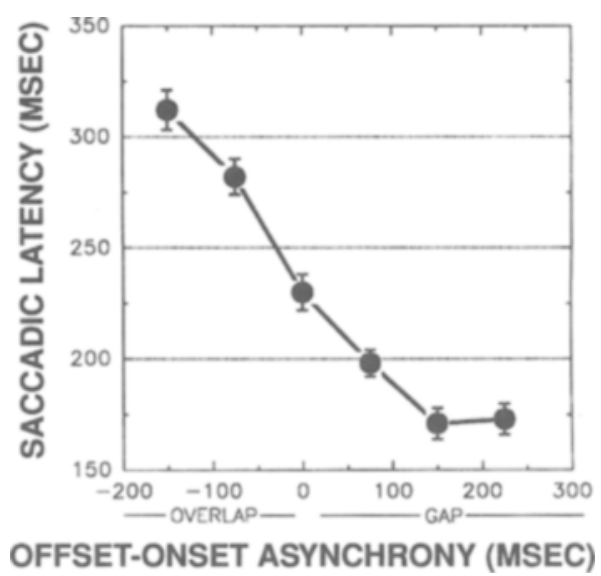

Figure 2. Saccadic latency as a function of the interval between the termination of an initially fixated stimulus and the appearance of a target for a saccade. Each circle represents the mean latency of five subjects. Vertical bars through the data points are standard errors of the mean. 
cute a manual rather than a saccadic response to the onset of the target. The purpose of this condition was to investigate whether the gap effect would generalize to manual responses.

\section{Method}

Subjects. Five subjects from the university community took part in the experiment. Four were experienced in eye-movement experiments.

Stimuli and Apparatus. Five light-emitting diodes, each $3 \mathrm{~mm}$ in diameter, were attached at intervals of $3^{\circ}$ to a metal rod. The rod was attached horizontally to the pen mount of an MFE 815M Plotomatic $x-y$ plotter. The middle diode was red in color and was used as a pursuit stimulus. The adjacent diodes, on either side of the middle diode, were green and were used as targets. The different colors for the pursuit stimulus and the targets helped the subjects readily identify the targets. The remaining outermost two diodes were used for calibration. The diodes were all in a frontal plane and were positioned $40 \mathrm{~cm}$ from the subject's corneal frontal plane when the subject looked straight ahead.

A Wavetek model 184 sweep generator was used to drive the pursuit and target stimuli with a sinusoidal frequency of $0.05 \mathrm{~Hz}$ and an amplitude of $24^{\circ}$, centered in the median plane of the subject. The resulting stimulus velocity was $2.4 \%$ sec. These frequency and velocity parameters were chosen because they were optimal for smooth pursuit (see Lisberger, Morris, \& Tychsen, 1987, p. 99).

The same system as described in Experiment 1 was used to monitor and record eye positions. For the manual task, a switchbox with two push-buttons was used. Buttonpresses were recorded, in conjunction with eye-position data, and the data were later transferred onto polygraphs for analysis.

Design. There were two factors in the experiment: (1) offset- onset condition, at two levels: overlap or gap; and (2) response task, at two levels: saccadic or manual. Thus, there were four experimental conditions ( 2 offset-onset conditions $\times 2$ response tasks). Note that with respect to the pursuit stimulus, it either remained on (overlap condition) or was turned off simultaneously with target appearance ("gap" condition). With respect to the response task, the subjects either changed fixation to the target or pressed one of two push-buttons (l $\mathrm{cft} / \mathrm{right}$ ) corresponding to the target (left/right) that appeared during visual pursuit.

Procedure. At the beginning of a trial, the subjects visually pursued a moving stimulus that was the only visible stimulus in an otherwise dark room. The subjects responded to a target that appeared either to the left or to the right of the moving stimulus. They were instructed to respond to the target as quickly as possible. In the saccadic task, the subjects moved their eyes to the target; in the manual task, they pressed a button on the side of the switchbox that corresponded to the side on which the target appeared, with respect to the pursuit stimulus. The target was turned on, after a random delay, only if a computer detected that eye position was within the central $15^{\circ}$ portion of the full amplitude of pursuit movement, which was $24^{\circ}$.

Each subject completed 80 trials per experimental condition (the total number of trials, irrespective of target position; no systematic latency differences were observed with latencies obtained with the left and the right target during leftward or rightward pursuit movements.) The trials were divided into four sessions, each conducted on a separate day. Each session consisted of four 20-trial blocks with random target positions either to the left or to the right of the moving stimulus. The first two blocks consisted of either the saccadic task or the manual task. The order of the tasks was counterbalanced among sessions. Within each block of trials, target position (left/right) and offset-onset condition (overlap/gap) were randomly selected.

\section{Results and Discussion}

Oculomotor latencies. The purpose of Experiment 2 was to examine latencies of saccades to a target while the eyes were engaged in smooth pursuit. Figure 3 shows the mean saccadic latencies averaged over all five subjects (filled circles). Latencies were shorter when the pursuit stimulus was turned off on target onset (gap condition) than they were when it was left on (overlap condition). Saccadic latencies were reduced by an average of $68 \mathrm{msec}$ in the gap condition (Tukey's $H S D=65.07$, $p<.01$ ). Recently, we have become aware of an abstract reporting similar findings (Frecker, MacLean, Eizenman, \& Collis, 1991).

The patterns of results obtained in the present experiment using a moving fixation stimulus and in those reported by others using a stationary fixation stimulus (e.g., Becker, 1972; L.E. Ross \& S. M. Ross, 1980; Saslow, 1967) emphasize the functional similarity between the tasks of pursuit and visual fixation. In both cases, the goal is to stabilize the image of a stimulus on the fovea. The present results suggest that turning off the stimulus leads to disengagement of pursuit or fixation, thus enabling the oculomotor system to respond more readily to a new target. Note, however, that the functional similarity of pursuit and fixation does not imply that they are identical in every respect (see Luebke \& Robinson, 1988).

Manual latencies. In contrast to the results for saccadic latencies, manual latencies were not significantly different between gap and overlap conditions. On average, in gap conditions, latencies were shorter by $17 \mathrm{msec}$ than they were in overlap conditions. The open triangles in Figure 3 show the mean manual latencies for all five subjects.

The contrasting results of manual latencies and saccadic latencies were confirmed with an analysis of variance. There was a statistically significant interaction

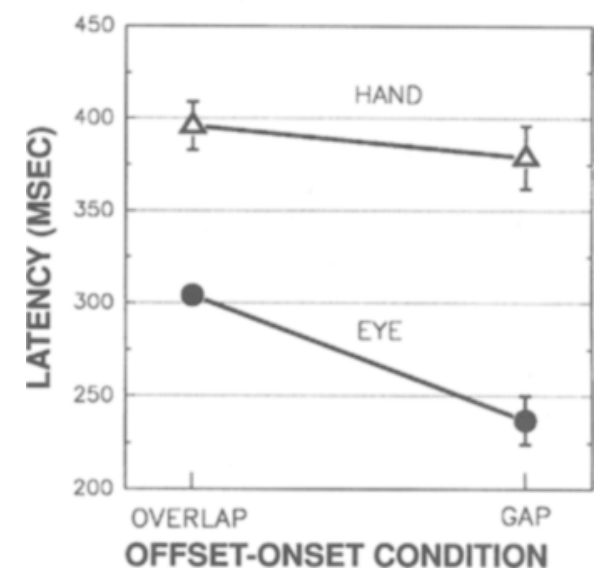

Figure 3. Response latency as a function of whether a visually pursued stimulus was left unchanged (overlap condition) or was turned off at the same time as the appearance of a target for a saccade (gap condition). Each circle and triangle represents the mean latency of five subjects. Vertical bars through the data points are standard errors of the mean. 
between response task (oculomotor/manual) and offsetonset condition (gap/overlap) on response latency $[F(1,4)$ $=13.01, p<.05]$. These results suggest that the gap effect observed in the present experiment was specific to the oculomotor task. The results may be readily explained by the fact that the oculomotor task required oculomotor disengagement, whereas the manual task did not. In the oculomotor task, the subjects had to suspend engagement of fixation during smooth pursuit before a saccade to the new target. In the manual task, however, the subjects did not have to disengage fixation to press a button in response to target onset. Thus, a facilitation of oculomotor disengagement in gap conditions would have contributed to reduced latencies in the oculomotor task, but would not have done so in the manual task. Frequency distributions of saccadic latencies, for a representative subject, are shown in Figure A2 in the appendix.

\section{EXPERIMENT 3}

This experiment extended the investigation of the gap effect to extrafoveal fixation stimuli. Saccadic latencies to targets appearing during extrafoveal fixation were measured. For comparison, we also measured saccadic latencies during foveal fixation. In addition, a manual task was included in both foveal and extrafoveal fixation tasks.

Normally, during visual fixation, the image of a stimulus is maintained on the fovea. During extrafoveal fixation, the image of the stimulus is maintained on the peripheral retina (Bedell, Barbeito, \& Aitsebaomo, 1984; Rattle, 1969). As with foveal fixation, the goal of extrafoveal fixation is to maintain the image of a fixated stimulus relatively motionless on the retina. According to the oculomotor disengagement hypothesis, turning off the extrafoveal fixation stimulus before target onset (gap condition) should facilitate disengagement by freeing the oculomotor system to respond to the target, thereby reducing saccadic latencies to the target.

\footnotetext{
Method

Subjects. Five subjects with normal ocular mobility were recruited from the university community. Two were new to eyemovement experiments.

Stimuli and Apparatus. Stimuli were light-emitting diodes, similar to those used in the previous experiments. The extrafoveal fixation stimulus consisted of two diodes that were located $6^{\circ}$ apart along an imaginary vertical line, in the median plane of a subject. The foveal fixation stimulus was a diode located at the midpoint between the two vertically spaced diodes. The foveal fixation stimulus was at eye level and was located $60 \mathrm{~cm}$ from the subject. As targets for saccades, one diode was located $3^{\circ}$ to the left and another $3^{\circ}$ to the right of the foveal fixation stimulus. For calibration, additional diodes were placed $6^{\circ}$ on either side of the foveal stimulus. The timing of the onset and the offset of the diodes was controlled by a computer.

The switchbox with the push-buttons for manual responses, the bite bar for stabilizing the head, and the eye-monitoring and recording system were those used in Experiment 2. The analog eyeposition data were sampled at $100 \mathrm{~Hz}$ with an $\mathrm{A} / \mathrm{D}$ converter (AI02 from Interactive Structures Inc.). Saccades were detected by the computer, using a velocity criterion of $25 \% \mathrm{sec}$, as used by other
}

investigators (c.g., Hallett \& Adams, 1980). Buttonpresses were also detected by the computer.

Design. There were three factors in the experiment: (1) response task, at two levels: saccadic or manual; (2) fixation condition, at two levels: extrafoveal or foveal; and (3) offset-onset asynchrony, at two levels: $-150 \mathrm{msec}$ (overlap condition) and $100 \mathrm{msec}$ (gap condition). Thus, there were eight experimental conditions $(2$ response tasks $\times 2$ fixation conditions $\times 2$ offset-onset asynchronies).

Procedure. The experiment was conducted in a room that, apart from the light emitted by the diodes, was completely dark. At the beginning of a trial, either the two vertically spaced diodes or the diode at eye level was lit. If the two vertically spaced diodes were on, the subjects fixated the midpoint between them (extrafoveal fixation). If the single diode was on, the subjects fixated the diode (foveal fixation). As in Experiment 2, the subjects were instructed to respond as quickly as possible to target onset with either a saccadc or a buttonpress.

Each subject completed 80 trials per experimental condition. The trials were completed in two sessions, each on a separate day. A session consisted of eight consecutive 20-trial blocks for the saccadic task and another eight consccutive 20-trial blocks for the manual task. The order of the response tasks was counterbalanced across subjects and across sessions. Target position, fixation condition, and offset--onset asynchrony were randomly varied within cach block of trials. The foreperiod before target onset was also varied from trial to trial.

\section{Results and Discussion}

Oculomotor latencies. The purpose of Experiment 3 was to explore the gap effect with extrafoveal fixation. According to the oculomotor-disengagement explanation of the gap effect, turning off the extrafoveal stimulus should facilitate disengagement and should help reduce saccadic latencies to a target. Thus, when the extrafoveally fixated stimulus is turned off before target onset, saccadic latencies might be expected to be shorter than they are when it is turned off after target onset.

The mean data for all five subjects are shown in Figure 4. The upper panel shows the data for extrafoveal fixation and the lower panel the data for foveal fixation.

For extrafoveal fixation, the mean difference in saccadic latency between the gap condition and the overlap condition was $52 \mathrm{msec}$. In the upper panel of Figure 4 , note that the filled circle in the gap condition is below that in the overlap condition. This difference was statistically significant (Tukey's HSD $=20.75$, $p<.01$ ). Frequency distributions of saccadic latencies, for a representative subject, are shown in Figure A3 in the appendix.

For foveal fixation, a gap effect for saccadic latencies was also observed. This result is consistent with the findings of Experiment 1 and with those reported by previous researchers (e.g., L. E. Ross \& S. M. Ross, 1980; Saslow, 1967; and many others). The results of foveal fixation are shown in the lower panel of Figure 4. Note that the filled circle in the gap condition is below that in the overlap condition. The mean difference in saccadic latency between the gap condition and the overlap condition was $81 \mathrm{msec}$ (Tukey's $H S D=20.75, p<.01$ ).

The most important aspect of the findings of the present experiment was that the gap effect was also observed 


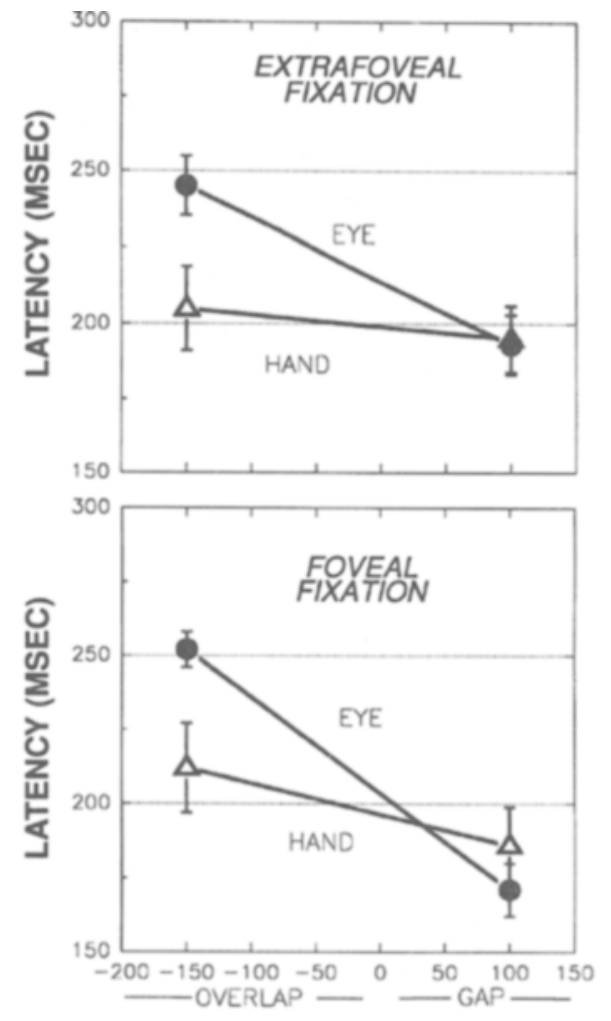

OFFSET-ONSET ASYNCHRONY (MSEC)

Figure 4. Response latency as a function of the interval between the termination of an initially fixated stimulus and the appearance of a target for a response. The top panel shows the data obtained with an extrafoveal fixation stimulus, while the bottom panel shows the data obtained with a foveal fixation stimulus. Each circle and triangle represents the mean latency of five subjects. Vertical bars through the data points are standard errors of the mean.

with extrafoveal fixation. This suggests that fixation is not restricted to visual processing at the fovea and that events leading to the execution of a saccade are similar, whether the initially fixated stimulus is located extrafoveally or foveally.

Manual latencies. In contrast to saccadic latencies, manual latencies in gap conditions did not show a significant reduction compared with those in overlap conditions. This pattern of results concurred with that obtained in Experiment 2, although absolute manual latencies were shorter in Experiment 3, possibly because in the latter, the subjects did not have to pursue the fixation stimulus. The absence of a gap effect for manual latencies suggests that the gap effect is specific to the oculomotor task. This pattern of results is consistent with the notion that oculomotor disengagement is required prior to executing an oculomotor response, but not prior to executing a manual response.

The absence of a significant gap effect with manual latencies indicated that warning did not play a major role in producing the gap effect in the present experiment. In the gap condition, turning off the fixation stimulus before target onset could have warned the subjects of the impending target (e.g., L. E. Ross \& S. M. Ross, 1980). In the overlap condition, on the other hand, there was no warning of the impending target. An effect of warning would be manifested in shorter latencies in gap conditions than in overlap conditions. However, an effect of warning was not observed in the manual latency data. This implies that effects of warning were minimal in the present experiment. Note that the onset of the target was not easily predicted because target position, offset-onset asynchrony, and the foreperiod before target onset were varied randomly, from trial to trial. Absence of a gap effect for manual latencies was also reported by ReuterLorenz et al. (1991).

Nevertheless, effects of warning may have played a more significant role in a number of other studies, in which manual latencies were significantly shorter in gap conditions than they were in overlap conditions (e.g., Iwasaki, 1990; Tam \& Stelmach, 1993). However, even in those studies, the gap effect was larger for the oculomotor task (saccade) than it was for the manual task (buttonpress). This interaction between response task (saccade/buttonpress) and offset-onset asynchrony (overlap/gap) on response latency was confirmed in the present study $[F(1,4)=39.23, p<.01]$. The overall pattern of results suggests to us that effects of oculomotor processes occur over and above any effect due to warning. This interpretation concurs with the conclusions of Kingstone and Klein (1993a) and Reuter-Lorenz et al. (1991).

\section{GENERAL DISCUSSION}

Previous studies of the gap effect have focused on latencies of saccades to targets appearing during foveal fixation (e.g., Becker, 1972; Cohen \& Ross, 1977; Fischer \& Ramsperger, 1984; Iwasaki, 1990; Reulen, 1984; Reuter-Lorenz et al., 1991; L. E. Ross \& S. M. Ross, 1980; S. M. Ross \& L. E. Ross, 1981, 1983; Saslow, 1967). It was found that when the foveated stimulus was turned off before target onset, saccadic latencies were shorter than they were when the foveated stimulus was left on.

The present study extended the response and stimulus domains of studies of the gap effect. The gap effect was found for vergence eye movements during foveal fixation (Experiment 1), and for saccadic latencies during smooth pursuit of a moving stimulus (Experiment 2) and during extrafoveal fixation (Experiment 3 ).

A common denominator of the different oculomotor tasks examined in the present study was the incompatibility between the response required by the fixation stimulus and the response required by the new target. Whereas the initial stimulus required a fixation-locking response, the new target required a fixation-shifting response. The goal of the fixation-locking response (fixation, pursuit, or extrafoveal fixation) was to keep the image of the fixation stimulus relatively motionless on the retina. In contrast, the goal of the fixation-shifting response (vergence and saccades) was to move the retinal image of the target across the retina onto the fovea. 
Since the oculomotor system could not respond to both goals at the same time, fixation locking would have to be suspended or disengaged to permit a shift in fixation to occur. A gap effect was manifested because removing the fixation stimulus eliminated the response incompatibility between fixation locking and fixation shifting, thereby facilitating disengagement. In turn, this facilitation reduced latencies in gap conditions compared with those in overlap conditions. Klein (1978), in proposing one of two possible interpretations of the gap effect, also suggested elimination of response competition in gap conditions as a possible explanation of the effect.

The notion of oculomotor disengagement is echoed in the work of other researchers. For example, Lynch et al. (1977) suggested that foveal fixation must be "broken momentarily" to allow for rapid shifts in fixation. Their suggestion was based on the observation that the activity of visual fixation cells in the parietal lobe of macaques is suppressed during or even before a saccadic movement, "pari passu with the incremented activity of the saccade neurons" (p. 376). Hallett and Adams (1980) suggested that "survival requires that there be a goalselecting mechanism whereby other contingencies can free the gaze from the 'stimulus,' otherwise visual search of many scenes would be impossible, and the saccadic servomechanism would lock the gaze onto a single conspicuous feature" (p. 331). S. M. Ross and L. E. Ross (1981, p. 435), in their study of different types of warning signals on saccadic latency, hypothesized that either fixation stimulus disappearance or a peripheral target appearance may initiate "release" of the eyes from a visually fixated stimulus. Fischer and Ramsperger (1984) considered the possibility of a break of fixation as a necessary prerequisite for the next saccade, although they also considered the possibility of attentional disengagement. Mayfrank et al. (1986) suggested that the process of unlocking the eyes from a visually fixated stimulus consists of two processes, namely, "visuomotor" and "visuo-cognitive"; the visuo-motor process corresponds to oculomotor disengagement, and the visuo-cognitive process to attentional disengagement.

The potential value of the current approach, which emphasizes the response incompatibility between fixation locking and fixation shifting, is in providing a general framework for studying oculomotor latencies and the gap effect. For example, the issue of whether a gap effect occurs for antisaccades (e.g., Fischer \& Weber, 1992; Reuter-Lorenz et al., 1991) can be approached with this framework in mind. Instead of asking whether a gap effect occurs for antisaccades, one might ask under what stimulus conditions a gap effect would occur for antisaccades. In an antisaccade task, a subject fixates a stimulus located centrally and shifts fixation to an imaginary target when a stimulus appears in the periphery. The imaginary target is located at the same distance from initial fixation as the peripheral stimulus is, but on the other side of stimulus appearance. Given the current framework, incompatibilities may be noted among three oculomotor tasks or programs: (1) the program main- taining visual fixation; (2) the program for a reflexive eye movement to the peripheral stimulus; and (3) an internally generated program to move the eyes to an imaginary target. If an experimental condition could be devised to minimize incompatibilities among the three tasks, it is possible that a gap effect might be observed. Thus, by focusing on response incompatibilities, the present study provides a general framework for approaching research issues dealing with oculomotor latencies and the gap effect.

In summary, the present research extended the task and stimulus domain in studies of the gap effect. The gap effect was demonstrated for vergence latencies during foveal fixation and for saccadic latencies during smooth pursuit and extrafoveal fixation. Considered together, the results indicate the generality of the gap effect for tasks requiring shifts in fixation. We conclude that the gap effect can be observed when a response required by target onset (e.g., saccade or vergence) is incompatible with that required by an initial stimulus (e.g., visual fixation, smooth pursuit, or extrafoveal fixation).

\section{REFERENCES}

BECKER, W. (1972). The control of eye movements in the saccadic system. Bibliotheca Ophthalmologica, 82, 233-243.

Bedel., H. E., Barbeito, R., \& Aitsebaomo, P. A. (1984). The precision of oculocentric direction and its role in the stability of fixation. Vision Research, 10, 1157-1161

Braun, D., \& Breitmeyer, B. G. (1988). Relationship between directed visual attention and saccadic reaction times. Experimental Brain Research, 73, 546-552.

Cohen, M. E., \& Ross, L. E. (1977). Saccadic latency in children and adults: Effects of warning interval and target eccentricity. Journal of Experimental Child Psychology, 23, 539-549.

FisCHER, B. (1987). The preparation of visually guided saccades. Re view of Physiology, Biochemistry, \& Pharmacology, 106, 1-35.

Fischer, B., \& Bretrmeyer, B. G. (1987). Mechanisms of visual attention revealed by saccadic eye movements. Neuropsychologia, 25, 73-78.

Fischer, B., \& RAmSPERGER, E. (1984). Human express saccades: Extremely short reaction times of goal directed eye movements. Experimental Brain Research, 57, 191-195.

FISCher, B., \& Weber, H. (1992). Characteristics of "anti" saccades in man. Experimental Brain Research, 89, 415-424.

Fischer, B., \& Weber, H. (1993). Express saccades and visual attention. Behavioral \& Brain Sciences, 16, 553-610.

Frecker, R. C., Maclean, W. J., Eizenman, M., \& Collis, R. (1991). Comparison of latencies of saccades elicited during fixation and smooth pursuit. Supplement to Investigative Ophthalmology \& Visual Science, 32, 1021

Guirton, D., Buchtel, H. A., \& Douglas, R. M. (1985). Frontal lobe lesions in man cause difficulties in suppressing reflexive glances and in generating goal-directed saccades. Experimental Brain Research, $\mathbf{5 8}, 455-472$.

Hallett, P. E., \& ADAMs, B. D. ( 1980 ). The predictability of saccadic latency in a novel voluntary oculomotor task. Vision Research, 20, 329-339.

IWASAKI, S. (1990). Facilitation of reaction times with gap paradigm: Comparison of manual and saceadic responses. Ergonomics, 33, 833-850.

Kincistone, A., \& KLEIN, R. M. (1993a). Visual offsets facilitate saccadic latency: Does predisengagement of visuospatial attention mediate this gap effect? Journal of Experimental Psychology: Human Perception \& Performance, 19, 1251-1265.

Kingsione, A., \& KLEIN, R. M. (1993b). What are human express saccades? Perception \& Psychophysics, 54, 260-273. 
KLEIN, R. (1978). Chronometric analysis of saccadic eye movement: Reflexive and cognitive control. In D. Landers \& R. Christina (Eds.). Psychology of motor behavior and sport 1977 (pp. 247-254). Champaign. IL: Human Kinetics.

Klein, R., Kingstone, A., \& Pontefract, A. (1992). Orienting of visual attention. In K. Rayner (Ed.), Eye movements and visual cognition: Scene perception and reading (pp. 46-65). New York: Springer-Verlag.

Lisberger, S, G., Morris, E. J., \& Tychsen, L. (1987). Visual motion processing and sensory-motor integration for smooth pursuit eye movements. Annual Review of Neuroscience, 10, 97-129.

Luebke, A. E., \& Robinson, D. A. (1988). Transition dynamics between pursuit and fixation suggest different systems. Vision $R e$ search, 28, 941-946.

Lynch, J. C., Mountcastle, V. B., Talbot, W. H., \& Yin, T. C. T. (1977). Parietal lobe mechanisms for directed visual attention. Journal of Neurophysiology, 40, 362-389.

Marocco, R. T. (1978). Saccades induced by stimulation of the frontal cye fields: Interaction with voluntary and reflexive eye movements. Brain Research, 146, 23-34.

Mayfrank, L., Mobashery, M., Kimmig, H., \& Fischer, B. (1986). The role of fixation and visual attention in the occurrence of express saccades in man. European Archives of Psychiatry \& Neurological Sciences, 235, 269-275.

RATTLE, J. D. (1969). Effect of target size on monocular fixation. $O p$ tica Acta, 16, 183-192.

RelleN, J. P. H. (1984). Latency of visually evoked saccadic eye movements: I. Saccadic latency and the facilitation model. Biological Cybernetics, 50, 251-262.

Relter-Lorenz, P. A., Hughes, H. C., \& Fendrich, R. (1991). The reduction of saccadic latency by prior offset of the fixation point: An analysis of the gap effect. Perception \& Psychophysics, 49, 167-175.

Ross, L. E., \& Ross, S. M. (1980). Saccade latency and warning signals: Stimulus onset, offset, and change as warning events. Perception \& Psychophysics, 27, 251-257.

Ross, S. M., \& Ross, L. E. (1981). Saccade latency and warning signals: Effects of auditory and visual stimulus onset and offset. Perception \& Psychophysics, 29, 429-437.

Ross, S. M., \& Ross, L. E. (1983). The effects of onset and offset warning and post-target stimuli on the saccade latency of children and adults. Journal of Experimental Child Psychology, 36, 340-355.
SAsLow, M. G. (1967). Effects of components of displacement-step stimuli upon latency for saccadic eye movements. Journal of the Optical Society of America, 57, 1024-1029.

TAM, W. J., \& STELMACH, L. B. (1993). Viewing behavior: Ocular and attentional disengagement. Perception \& Psychophysics, 54, 211 222.

Wenban-Smith, M. G., \& Findlay, J. M. (1991). Express saccades: Is there a separate population in humans? Experimental Brain Research, 87, 218-222.

Zuber, B. L., \& STARK, L. (1968). Dynamical characteristics of the fusional vergence eye movement system. IEEE Transactions on Systems, Science \& Cybernetics, 4, 72-79.

\section{APPENDIX}

In the present research, we were interested in the gap effect and not in the occurrence of bimodality/express saccades in latency distributions. The occurrence or nonoccurrence of bimodality/express saccades is a separate issue that is still under debate (Fischer \& Weber, 1993; Kingstone \& Klein, 1993a; Reuter-Lorenz et al., 1991; Wenban-Smith \& Findlay, 1991). Nevertheless, for those interested in the issue, representative latency distributions from Experiments 1, 2, and 3 are provided in Figures A1, A2, and A3, respectively. In Figure A1, each histogram comprises 20-30 observations for vergence latencies and 40-50 observations for saccadic latencies. In Figures A2 and A3, each histogram comprises 80 observations. Weak evidence of bimodality/express saccades is observed in some of the histograms. Specifically, bimodality/express saccades are observed only for saccadic latencies in the $+75 \mathrm{msec}$ and $+150 \mathrm{msec}$ gap conditions of Figure Al. Bimodality/express saccades have been reported more reliably by Fischer and colleagues (see Fischer \& Weber, 1993), although not by others (Kingstone \& Klein, 1993b; Reuter-Lorenz et al., 1991; Tam \& Stelmach, 1993; Wenban-Smith \& Findlay, 1991).

More important to the goal of the present study, the gap effect evident in the mean data shown in Figures 1, 2, 3, and 4 is also evident in the latency distributions of Figures A1, A2, and A3. Note that, compared with overlap conditions, the distributions are shifted to the left for gap conditions. 


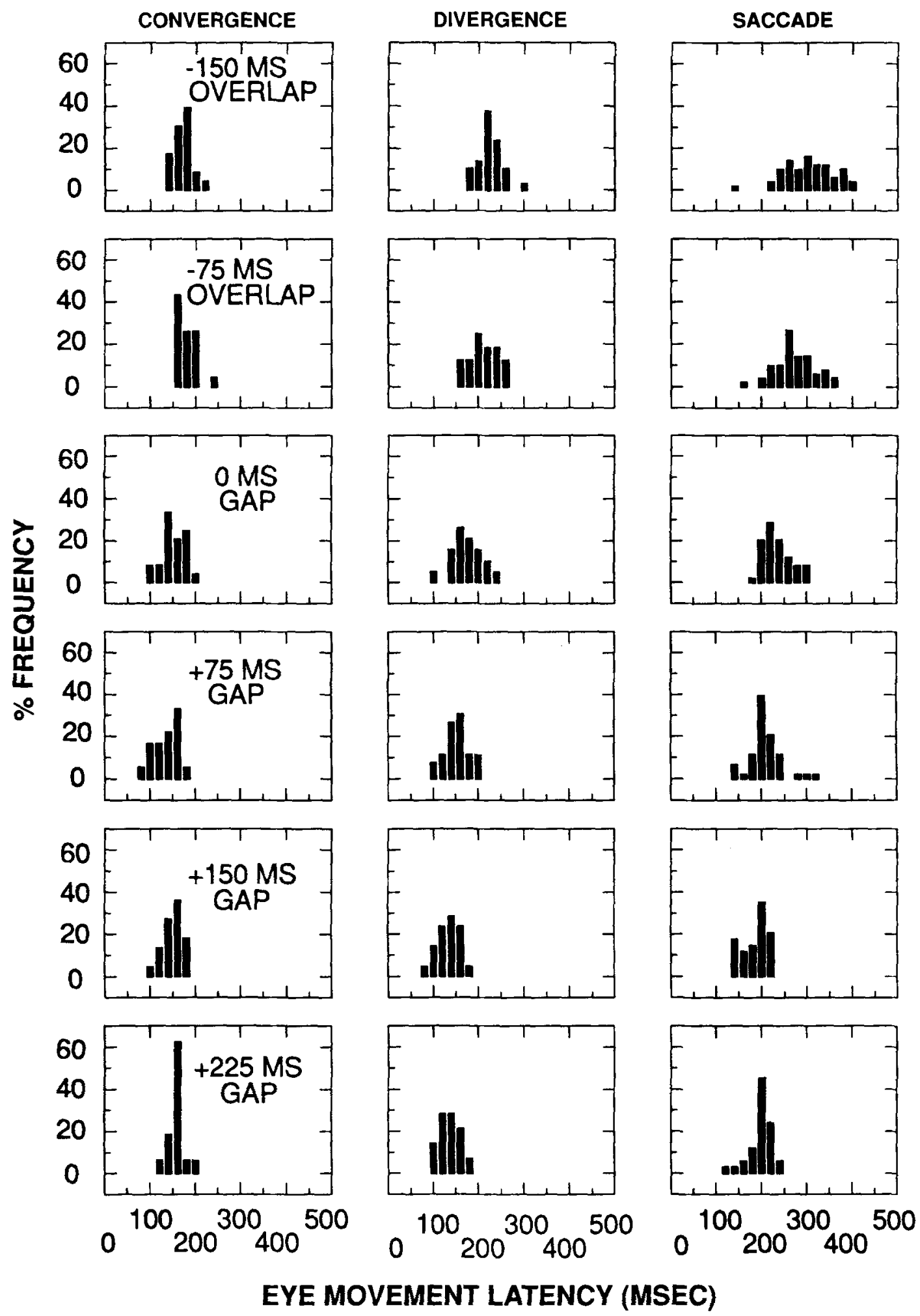

Figure A1. Frequency distributions of vergence and saccadic latencies for a representative subject, for different temporal asynchronies between fixation stimulus offset and target onset. Bin width is 20 msec. 

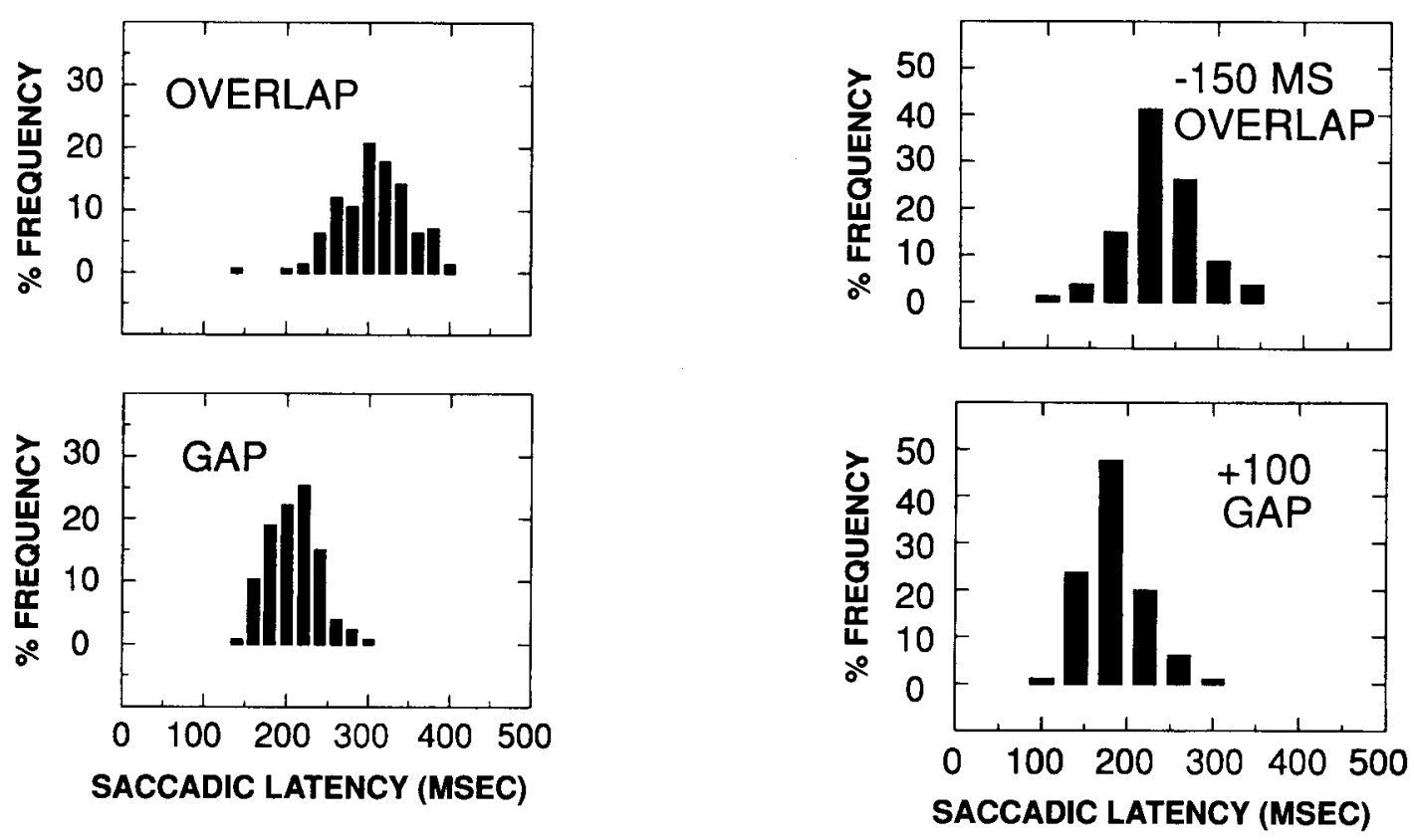

Figure A2. Frequency distributions of latencies of saccades during visual pursuit for a representative subject. Bin width is 20 msec. The data for the top panel were obtained with the pursued stimulus left unchanged on target onset (overlap condition). The data for the bottom panel were obtained with the pursued stimulus turned off on target onset (gap $=0$ msec condition).

Figure A3. Frequency distributions of latencies of saccades during extrafoveal fixation for a representative subject. Bin width is $40 \mathrm{msec}$.

(Manuscript received August 28, 1992;

revision accepted for publication March 21, 1994.) 